\title{
Input-output efficiency of 20 independent research teams at State Key Laboratory of Respiratory Disease 2009-2016: managerial implications for elsewhere in China
}

\author{
Rui Bao ${ }^{1}$, Hai-Shang Zhuang ${ }^{1}$, Wei Peng ${ }^{2}$, Ming Li $^{2}$, Kai-Ping Li $^{2}$, Lin Zhou ${ }^{3}$, Tian-Qiang Liu ${ }^{2}$, \\ Xin-Wang Wang ${ }^{4}$ \\ ${ }^{1}$ Department of Epidemiology and Health Statistics, School of Public Health, ${ }^{2}$ Department of Research, ${ }^{3}$ Department of Propaganda, ${ }^{4}$ School of \\ General Medicine and Continuing Education, Guangzhou Medical University, Guangzhou 510182, China \\ Correspondence to: Xin-Wang Wang, MD, PhD. School of Public Health, Guangzhou Medical University, No. 195, Dong Feng West Road, \\ Guangzhou 510182, China. Email: xinwwang@aliyun.com.
}

Submitted Oct 16, 2018. Accepted for publication Dec 10, 2018.

doi: $10.21037 /$ jtd.2018.12.61

View this article at: http://dx.doi.org/10.21037/jtd.2018.12.61

Currently, China's State Key Laboratories (SKLs) play a vital role in the trend of scientific innovation in this amazing country. Specifically, SKLs as essential bases for national high-level research and talent training, are crucial in improving the country's independent innovation capacity, core competitiveness, scientific and technical breakthrough, thereby leading China from a manufacturing to an innovating power. In this setting, analysis of the scientific input-output efficiency (IOE) may offer suggestion for decision-making in terms of funds optimization and management. An objective and reasonable approach of such analysis so far remains challenging, in light of so many factors associated with the scientific input-output issues. This can be shown in a recent attempt to look at the IOE of 20 independent research teams in State Key Laboratory of Respiratory Disease (SKLRD).

To start with, a round-table meeting of investigators and statisticians was summoned and led to decision on using data envelopment analysis (DEA) model (1) and Malmquist productivity index (MPI) as investigatory tools for IOE evaluation. The MPI reflects the dynamic change of the total factor productivity (TFPCH), and its efficiency varies. In the model, TFPCH comprises four parts: technical efficiency change $(\mathrm{EFFCH})$ to denote the change in management level and improvement in efficiency; technological change (TECHCH), the degree of technological progress; scale efficiency change (SECH), whether the operation of the team is at the optimal scale (2); and pure technical efficiency change (PECH) (3), the degree of technical level.

Given the principles of availability, scientificity, importance, representation, comparability $(4,5)$, and to practice in consistency with previous studies (6), three input variables (scientific research funds, scores of scientific research projects, and number of researchers) and five output variables (patents score, monographs score, graduated students score, research awards score, and publication impact factor) were selected according to expert recommendations. All the variables were calculated as described by Liao et al. (6).

To proceed with, 8-year panel data (2009 through 2016) were collected from the Guangzhou Medical University Scientific Research Administration and SKLRD. The average overall 8-year efficiency, pure technical efficiency, and scale efficiency were $0.789,0.902$, and 0.871 , respectively. The overall efficiency of individual team was not stable over years. The difference between the pure technical efficiency and scale efficiency was not too significant, but the reduction in the overall efficiency was primarily attributed to the low-scale efficiency $(0.871)$.

Besides, input redundancy and output insufficiency of research teams had to be evaluated. Regarding the input, all research teams at SKRLD from 2009 to 2016 year had to spend an average of 20 research teams a year with a total of $\leq 25$ researchers, 4.5463 million Yuan RMB less costs, and reduce research scores by 47.137 . Considering the output, 56.253 points to patents, 5.066 points to monographs, 15.220 to the number of graduate students, 14.324 points 
to research awards score, and 52.617 points to the impact factor score should be added.

From 2009 to 2016, the geometric mean of TFPCH reached 1.103, suggesting an average annual increase of $10.3 \%$. In addition, the TECHCH was 1.072 and the EFFCH was 1.029 , demonstrating that the technological progress and innovation were the major driving force of the overall efficiency improvement. Moreover, the improvement of technical efficiency promoted the TFPCH; however, its effect was relatively modest compared with technological progress $(2.9 \%)$, in which the contribution of $\mathrm{PECH}$ and SECH was $1.4 \%$ and $1.5 \%$, respectively. Although the overall efficiency was in a good development trend during 2009-2016, the TFPCH and decomposition efficiency change fluctuated, exhibiting a U-shaped tendency.

In this occasion, the overall efficiency is the total factor productivity which also implies the input-output efficiency. As far as our IOE analysis was concerned, it comprehensively reflects utilization efficiency of scientific research resources, ability to optimize the allocation of the research resources, and the management level. While the SOIE from 2009 to 2016 was 0.789 , the year 2013 saw the lowest value (0.685) compared with the others (0.751-0.874). This implied the room for improvement in the utilization efficiency and optimal allocation of scientific research resources in the 20 research teams so that the research teams can approach or even reach the current production frontier every year.

The pure technical efficiency denotes the production efficiency of the scientific research team affected by factors like management or technical level. The scale efficiency implies the difference between the existing size of the scientific research teams and the optimal size under certain management level and system. In the analysis, although the production level of the scientific research team is brought into play, the IOE was is not satisfactory because it does not reach the optimal production scale, that is, the investment of scientific research resources was wasted to varying degrees. In recent years, China has increased its support for scientific research institutions, demanding that the management ability of scientific research teams be further improved to match the scientific research resources. The investment should be neither wasted nor inadequate, so as to match optimally the situation of the team size.

It was found from the redundancy of input and insufficiency of output that the number of researchers does not represent the decisive factor of the high input-output efficiency. Under the guarantee of a good system, it is of great significance for the efficiency of scientific research input-output to give full play to the technical level of teams, improve the management ability and make the scientific research teams operate in the optimal scale.

The TFP of 20 independent SKRLD research teams exhibited a positive trend from 2009 to 2016, showing that the improvement of TFPCH efficiency is provided by EFFCH and TECHCH but primarily depends on the technological progress and innovation. Hence, achieving the optimal size and improving management capability remain the ongoing concerns for research teams.

Using DEA model and MPI method to analyze the efficiency of scientific research activities in SKLs can be of practical significance. First, the research efficiency in SKLs can appear more intuitively. Second, IOE evaluation may help SKL leaders identify the lowered scientific research efficiency caused by insufficient or excessive investment. Finally, to each research team, it could be conducive for assignment of scientific research activities, enhanced supervision and management, avoiding waste of resources caused by extensive input, and ultimately, achieving optimal resource allocation. We believe that the IOE evaluation attempts and experiences with SKLRD could be practically relevant in terms of scientific promotion for other SKLs in China.

\section{Acknowledgements}

None.

\section{Footnote}

Conflicts of Interest: The authors have no conflicts of interest to declare.

\section{References}

1. Coelli TJ, Rao DS, O'Donnell CJ, et al. An introduction to efficiency and productivity analysis. Springer Science \& Business Media, 2005.

2. Li RP, Wu JR, Cui HD. Evaluation and analysis to inputoutput efficiency of high tech industry in Hebei Province based on DEA. In: Proceedings of the International Conference on Machine Learning and Cybernetics (ICMLC'10). IEEE, 2010:1667-71.

3. Grilo A, Santos J. Measuring efficiency and productivity growth of new technology-based firms in business incubators: the Portuguese case study of Madan Parque. 
ScientificWorldJournal 2015;2015:936252.

4. Zhang Y, Wang Q, Jiang T, et al. Equity and efficiency of primary health care resource allocation in mainland China. Int J Equity Health 2018;17:140.

5. Cheng BH, Wang X, Pan T. Empirical study on the contribution rate of technological progress based on DEA-
Malmquist: Ten types of registered enterprises in Jiangsu Province in 2006-2015. J Civ Eng Manag 2018;35:179-86.

6. Liao JF, Wang J, Liang L, et al. An evaluation study of research efficiency of the Guangzhou institute of respiratory diseases based on malmquist index. J Thoracic Dis 2016;8:2709-16.
Cite this article as: Bao R, Zhuang HS, Peng W, Li M, Li KP, Zhou L, Liu TQ, Wang XW. Input-output efficiency of 20 independent research teams at State Key Laboratory of Respiratory Disease 2009-2016: managerial implications for elsewhere in China. J Thorac Dis 2018;10(12):6375-6377. doi: $10.21037 /$ jtd.2018.12.61 